\title{
Westerlund 1 as a Template for Massive Star Evolution
}

\author{
Ignacio Negueruela ${ }^{1}$, J. Simon Clark ${ }^{2}$, Lucy J. Hadfield ${ }^{3} \dagger$ and \\ Paul A. Crowther ${ }^{3}$ \\ ${ }^{1}$ Departamento de Física, Ingeniería de Sistemas y Teoría de la Señal, \\ Universidad de Alicante, Apdo. 99, E03080 Alicante, Spain \\ email: ignacio@dfists.ua.es \\ ${ }^{2}$ Department of Physics and Astronomy, The Open University, Walton Hall, \\ Milton Keynes MK7 6AA, United Kingdom \\ ${ }^{3}$ Department of Physics and Astronomy, University of Sheffield, \\ Sheffield, S3 7RH, United Kingdom
}

\begin{abstract}
With a dynamical mass $M_{\mathrm{dyn}} \sim 1.3 \times 10^{5} \mathrm{M}_{\odot}$ and a lower limit $M_{\mathrm{cl}}>5 \times 10^{4} \mathrm{M}_{\odot}$ from star counts, Westerlund 1 is the most massive young open cluster known in the Galaxy and thus the perfect laboratory to study massive star evolution. We have developed a comprehensive spectral classification scheme for supergiants based on features in the $6000-9000 \AA$ range, which allows us to identify $>30$ very luminous supergiants in Westerlund 1 and $\sim 100$ other less evolved massive stars, which join the large population of Wolf-Rayet stars already known. Though detailed studies of these stars are still pending, preliminary rough estimates suggest that the stars we see are evolving to the red part of the HR diagram at approximately constant luminosity.
\end{abstract}

Keywords. stars: early-type - stars: evolution - supergiants - stars: Wolf-Rayet - open clusters and associations: individual (Westerlund 1)

\section{Introduction}

As they evolve towards the Wolf-Rayet (WR) phase, massive stars must shed most of their outer layers. Models predict that massive stars will become supergiants (SGs) and evolve redwards at approximately constant $L_{\mathrm{bol}}$, but observations reveal a complex zoo of transitional objects, comprising Blue SGs, Red SGs, Yellow Hypergiants (YHGs), Luminous Blue Variables (LBVs) and OBfpe/WNVL stars, whose identification with any particular evolutionary phase is difficult. Understanding this evolution is, however, crucial because the mass loss during this phase completely determines the contribution that the star will make to the chemistry of the ISM and even the sort of post-supernova remnant it will leave.

Unfortunately, massive stars are scarce and, as this phase is very short on evolutionary terms, examples of massive stars in transition are rare. For most of them, distances are unknown and so luminosities are known at best to order-of-magnitude accuracy, resulting in huge uncertainties in other parameters $\left(M_{*}, R_{*}\right)$. The difficulty to place these objects in the evolutionary sequence is obvious.

The open cluster Westerlund 1 (Wd 1) offers an unprecedented opportunity to improve this situation. It contains a large population of evolved stars which have formed at the same time, are at the same distance and have the same chemical composition. With an age of 4-5 Myr, Wd 1 contains a rich population of stars in transitional states that can be used to constrain evolutionary models.

$\dagger$ Present address: Chester F. Carlson Center for Imaging Science, Rochester Institute of Technology, 54 Lomb Memorial Drive, Rochester NY, 14623, USA 


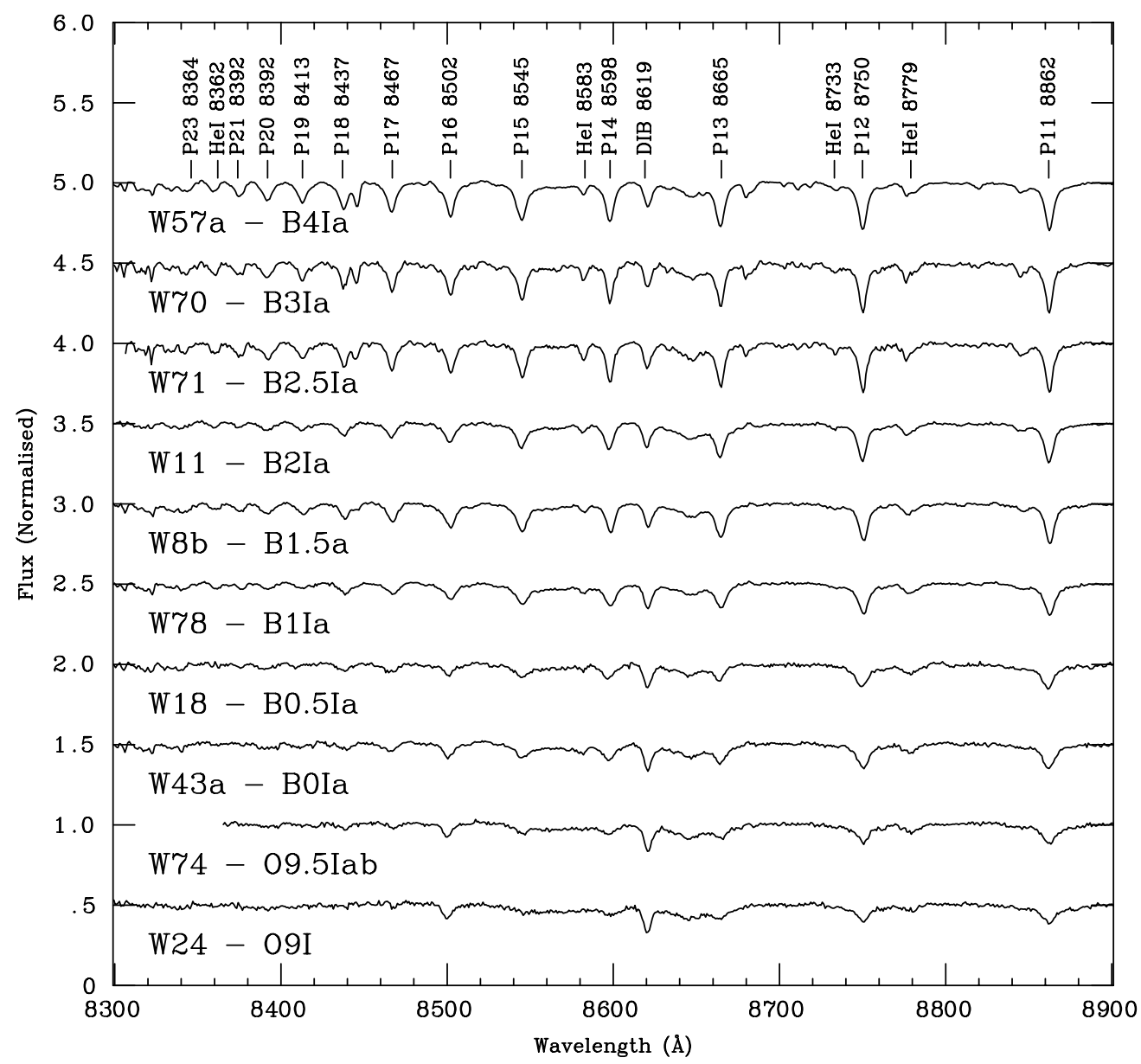

Figure 1. Sequence of $I$-band spectra of blue supergiants in $\mathrm{Wd} 1$, showing the evolution of the main features. Note the disappearance of $\mathrm{N}_{\mathrm{I}}$ features between $\mathrm{Pa} 12$ and $\mathrm{Pa} 13$ and $\mathrm{O}$ I $8446 \AA$ around $\mathrm{B} 2$ and the development of $\mathrm{Pa} 16$, which shows the presence of a strong $\mathrm{C}$ III line for stars B0 and earlier.

\section{Cluster parameters}

The parameters of $\mathrm{Wd} 1$ are still poorly determined, though significant progress has been made in recent years. Two estimates of its mass have been recently made. Mengel \& Tacconi-Garman (2008) have used the radial velocity dispersion of the ten stars brightest in the infrared $\left(\sigma=8.4 \mathrm{~km} \mathrm{~s}^{-1}\right)$ to estimate a mass of $\sim 1.3 \times 10^{5} \mathrm{M}_{\odot}$. This estimate suffers from a large uncertainty due to the low number of stars used and the spectral peculiarities of some. Brandner et al. (2008) have used star counts in the infrared to set a lower limit on the cluster mass $M_{\mathrm{cl}}>5 \times 10^{4} \mathrm{M}_{\odot}$. Again, there are important uncertainties coming into this estimation. Large discrepancies have been found in the determinations of distance and (hence) masses based on infrared pre-main-sequence tracks and optical postmain-sequence tracks. In any case, the integrated initial mass function appears consistent with Salpeter's and then the observed population of massive stars would imply a mass $\sim 10^{5} \mathrm{M}_{\odot}$, consistent with both determination. 
An important source of uncertainty in both measurements is the role of binarity, which affects both radial velocities and transformation of magnitudes into masses. Recent results suggest that the fraction of binaries with similar mass components is very high amongst cluster members. Most Wolf-Rayet stars show indirect indications of binarity, presence of dust in WC stars (Crowther et al. 2006) and hard X-ray emission in WN stars (Clark et al. 2008). Some of them also show photometric variability and at least one is an eclipsing binary (Bonanos 2007). The identification of a large number of X-ray sources detected by Chandra with a population of evolved late-O stars (Clark et al. 2008) suggests that the high binary fraction extends to lower masses.

The distance to the cluster is not very well constrained either. Photometry is affected by the very strong reddening $\left(A_{V} \approx 12 \mathrm{mag}\right)$. Analysis of the $E(B-V)$ colours suggests that the reddening deviates from the standard law (Clark et al. 2005). A recent determination, making use of atomic hydrogen in the direction to the cluster, gives $d=3.9 \pm 0.7$ (Kothes \& Dougherty 2007), compatible with, though slightly shorter than, estimates based on the stellar population (e.g., Crowther et al. 2006).

The age of the cluster can be constrained from the observed population. The ratio of WR stars to red and yellow hypergiants favours an age of 4.5 or $5.0 \mathrm{Myr}$, with the progenitors of the Wolf-Rayet stars having initial masses in the $40-55 \mathrm{M}_{\odot}$ range (Crowther et al. 2006). Such age is fully compatible with the observed population of blue supergiants (see below), which should be descended from stars with initial masses of $\sim 35 \mathrm{M}_{\odot}$. This again would imply masses of $\leqslant 30 \mathrm{M}_{\odot}$ for the stars at the top of the main sequence, in good agreement with the $\mathrm{O} 7-8 \mathrm{~V}$ spectral type, again appropriate for the age.

\section{Observations and analysis}

Observations of stars in Wd 1 were carried out on the nights of 2003 June 12th and 13th using the spectro-imager FORS2 on Unit 1 of the VLT (Antu) using three different modes: longslit, multi-object spectroscopy with masks (MXU) and multi-object spectroscopy with movable slitlets (MOS). We used grisms G1200R and G1028z to obtain intermediate resolution spectroscopy. With this setup, we obtained almost continuous coverage over the $5800-9500 \AA$ range at intermediate resolution.

Within the central $5^{\prime} \times 5^{\prime}$ field of view, we selected our targets from the list of likely members of Clark et al. (2005). For the external regions, targets were selected at random amongst relatively bright stars. In total, we took three MXU and one MOS mask with both G1200R and G1028z grisms, and two further MXU masks with only the G1200R (these were aimed at relatively faint objects, which were expected to be OB stars near the MS and so not to have strong features in the range covered by G1028z). This resulted in $\sim 100$ stars observed with G1200R and $\sim 70$ stars observed with G1028z. More than $90 \%$ of the spectra turned out to correspond to OB stars, and hence cluster members.

We have used these observations to derive spectral types for cluster members. The use of $I$-band spectra to classify OB stars has been explored by Caron et al. (2003) and is further discussed in Appendix A of Clark et al. (2005). We have studied further spectral type and luminosity indicators in the $R$ and $I$ bands and derived spectral types from a combination of features. Features that contribute to our analysis are: the shape and strength of emission (P-Cygni profiles) in $\mathrm{H} \alpha$, the strength of the $\mathrm{C}$ II $6578,6582 \AA$ doublet (in absorption), the presence and strength of the C II 7231, 7236 $\AA$ doublet (in emission, a wind feature), the strength of the O I $7774 \AA$ triplet and the strength of the N II $6482 \AA$ line. In the $I$ band, apart from the shape and strength of the Paschen lines (see Clark et al. 2005), a main indicator is the presence of the C III $8502 \AA$ line, which appears, blended into $\mathrm{Pa}$ 16, for stars B0 and earlier (see Fig. 1). 


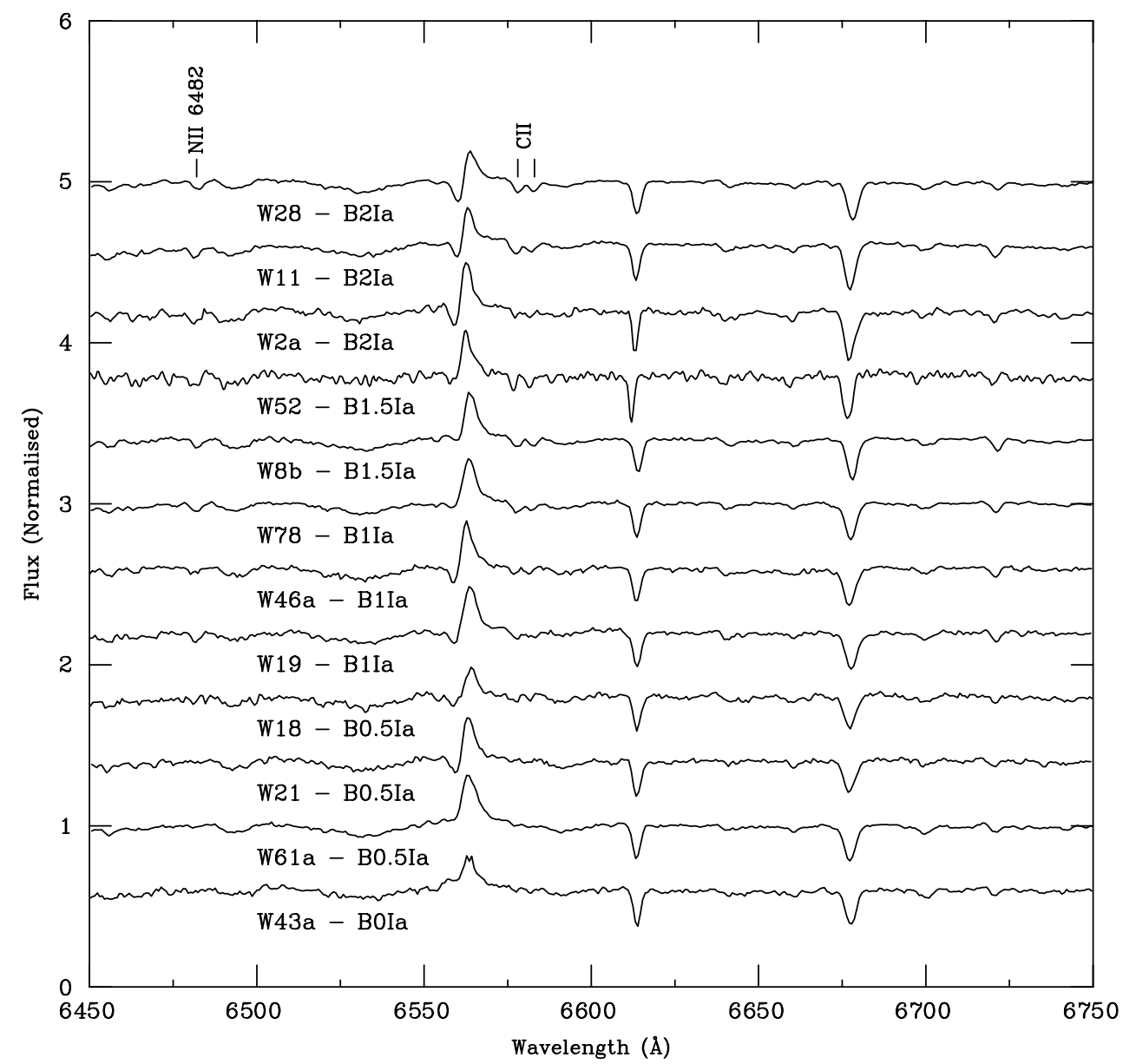

Figure 2. Sequence of red spectra of blue supergiants in $\mathrm{Wd} 1$. The shape of $\mathrm{H} \alpha$ changes from a P-Cygni profile to pure emission around spectral type B0.5. The $\mathrm{N}_{\text {II }} 6482 \AA$ line is only prominent in Ia supergiants and its strength peaks sharply at B2.

\section{Implications}

The upper HR diagram. The stellar content revealed by these observations is as follows. There is an A supergiant showing spectral variability (W243; Clark \& Negueruela 2004) and six other A/F hypergiants of very high luminosity, with $M_{V} \approx-9$ to -10 (Clark et al. 2005). We call these objects hypergiants because of their very high intrinsic magnitudes, without making any direct inference of their evolutionary status.

The same can be said of at least three late-B luminous stars: W42a (B8 $\left.\mathrm{Ia}^{+}\right), \mathrm{W} 33$ $\left(\mathrm{B} 5 \mathrm{Ia}^{+}\right)$and $\mathrm{W} 7\left(\mathrm{~B} 5 \mathrm{Ia}^{+}\right)$. In addition, there are $\sim 20$ supergiants that can be unambiguously classified as Ia, covering the B0 Ia-B4 Ia range. The earliest very luminous star that we find is W74, with spectral type O9.5 and a luminosity class that could be Ia or Iab.

Even though the exact luminosity class of O-type supergiants is more difficult to determine using red spectra, we do not find evidence for any other luminous O-type supergiant, though there are $>50$ and (taking into account the incompleteness of our dataset) likely 


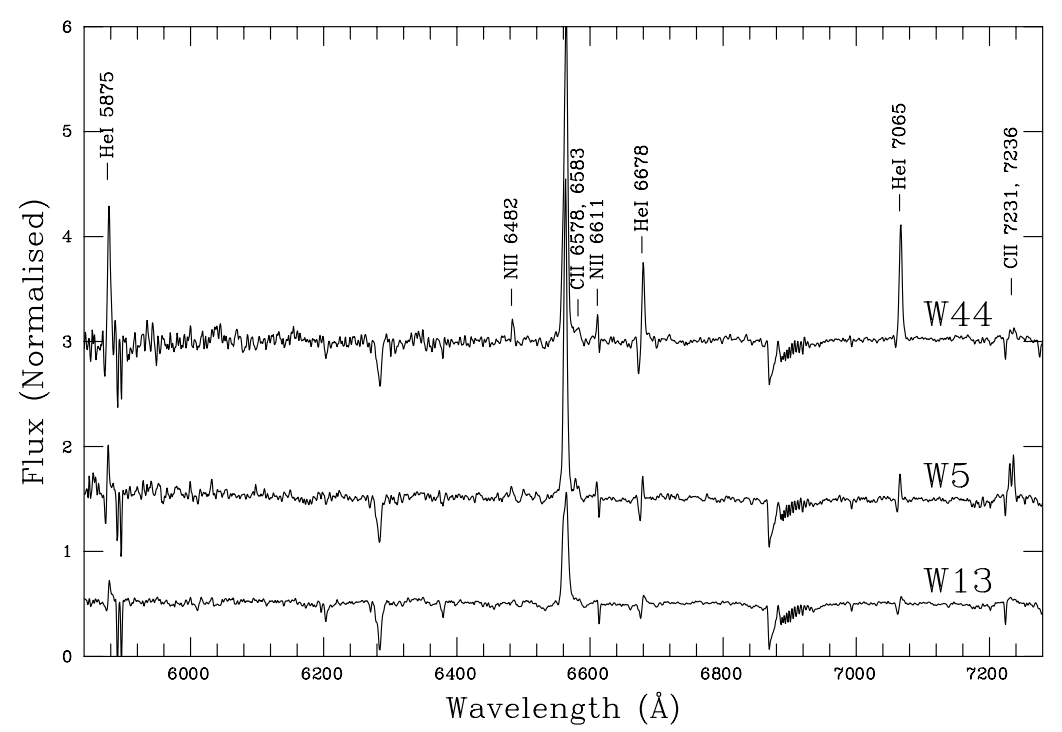

Figure 3. Spectra of three transitional objects in Westerlund 1, which can be classified as very late WN stars or early-B hypergiants. These objects might be examples of stars looping back to the blue region from the red supergiant phase.

$\sim 100$ stars which we generically classify as O9 I, meaning late-O stars well above the main sequence.

Evolution. We have made a rough attempt at calculating the intrinsic luminosities of all the supergiants, by using their $V$ and $I$ magnitudes and assuming that the relation between $E(V-I)$ and $M_{I}$ is standard. We use the intrinsic $(V-I)$ colours of Ducati et al. (2001) and bolometric corrections from Martins et al. (2005) and Humphreys \& McElroy (1984). Even though there is a large scatter, we find no clear trend. Stars of all spectral types seem to have similar bolometric magnitudes, a result consistent with the idea of redwards evolution at constant bolometric luminosity. Note that we have not compared the bolometric magnitudes of the red supergiants, as their spectral types are uncertain, and the bolometric corrections of such bright red stars are unknown.

This result does not imply that there are no stars looping back towards the blue in Wd 1. Obviously, the WR stars must have come back to the blue before reaching their present stage. There are three emission-line stars in the cluster which can be classified as very late WN stars or extreme B supergiants (see Fig. 3). These objects might be evolving into Wolf-Rayet stars. Detailed analysis of their spectra, in order to derive chemical abundances, will be necessary before their evolutionary status may be ascertained. Similar analyses should be conducted for the A and F hypergiants and W243.

The next steps in our work involve an investigation of the binary fraction in the cluster and detailed analysis of the spectra with state-of-the art models in order to obtain a better constraint on their evolutionary status.

\section{References}

Bonanos, A. Z. 2007, AJ, 133, 2696

Brandner, W., Clark, J. S., Stolte, A., et al. 2008, A\&SA, 478, 137

Caron G., Moffat A. F. J., St-Louis N., et al. 2003, AJ, 126, 1415

Clark J. S., Negueruela I., 2004, A\&3A, 413, L15 
Clark, J. S., Negueruela, I., Crowther, P. A., \& Goodwin, S. P. 2005, A\& A, 434, 949

Clark, J. S., Muno, M. P., Negueruela, I., et al. 2008, A\&A, 477, 147

Crowther, P. A., Hadfield, L. J., Clark, J. S., et al. 2006, MNRAS, 372, 1407

Ducati, J. R., Bevilacqua, C. M., Rembold, S. B., \& Ribeiro, D. 2001, ApJ, 558, 309

Humphreys, R. M. \& McElroy, D. B. 1984, ApJ, 284, 565

Kothes, R. \& Dougherty, S. M. 2007, A\&\&A, 468, 993

Martins, F., Schaerer, D., \& Hillier, D. J. 2005, A\&A, 436, 1049

Mengel, S. \& Tacconi-Garman, L. E. 2008, in: Vesperini, M. Giersz, \& A. Sills, (eds.), Dynamical Evolution of Dense Stellar Systems (Cambridge: CUP), Proc IAU Symp 246 in press (arXiv:0711.1779)

\section{Discussion}

LANG: What does the distribution of radio emission reveal in this cluster - both the extended emission and the point-like sources?

Negueruela: There are point sources associated with most of the cool and cold supergiants, which, we believe, are due to photoionisation of their extended atmospheres or winds by the hot stars. There is no extended emission obviously associated with the cluster, suggesting that supernova explosions have blown away any diffuse material. A more detailed summary may be found in the contribution by Dougherty \& Clark to Massive Stars: Fundamental Parameters and Circumstellar Interactions, (arXiv:0705.0971)

Crowther: As your title suggests, the presence of large numbers of massive stars in Wd 1 allows robust tests of evolutionary models. By way of example, $\mathrm{N}(\mathrm{WN}$ with $\mathrm{H})$ $<\mathrm{N}(\mathrm{WC})<\mathrm{N}(\mathrm{WN}$ without $\mathrm{H})$, yet both single (e.g., Geneva) and binary models (e.g., Eldridge et al. this volume) predict the completely wrong subtype distributions, namely $\mathrm{N}(\mathrm{WN}$ without $\mathrm{H})<\mathrm{N}(\mathrm{WN}$ with $\mathrm{H})<\mathrm{N}(\mathrm{WC})$ for an instantaneous burst of $4-5$ Myr.

Negueruela: Indeed. The observed population is most likely compatible with a single burst of star formation and hence offers an observational test of evolutionary models. Also, as I mentioned, the fact that we can observe a large population of massive $\left(M_{*}>\right.$ $\left.30 \mathrm{M}_{\odot}\right)$ stars evolving away from the main sequence and a very large population of low mass stars evolving towards the main sequence gives us an excellent opportunity to try to set post and pre-main-sequence isochrones in the same reference time and compare their predictions.

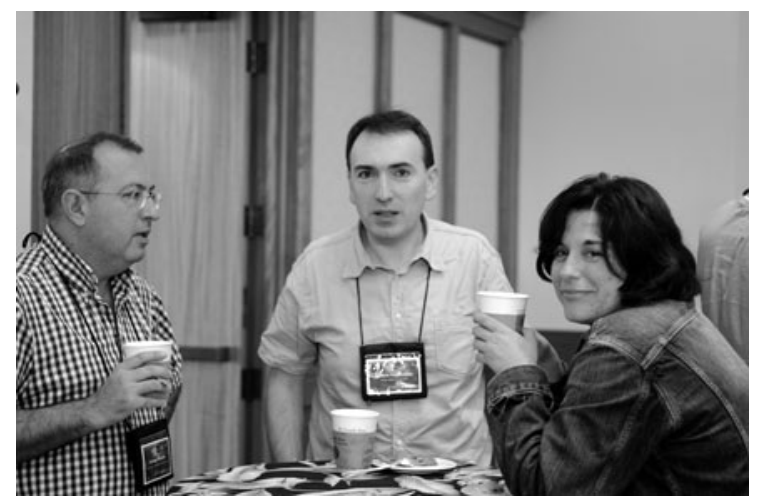

Ignacio Negueruela (center) with Amparo Marco (right) and Artemio Herrero (left). 